\title{
Diffusive benefits of cylinders in front of a Schroeder diffuser
}

\author{
M. A. Pogson, ${ }^{\text {a) }}$ D. M. Whittaker, and G. A. Gehring \\ Department of Physics and Astronomy, University of Sheffield, Sheffield S3 7RH, United Kingdom
}

\author{
T. J. Cox, R. J. Hughes, and J. A. S. Angus \\ Acoustics Research Centre, University of Salford, Salford M5 4WT, United Kingdom
}

(Received 17 February 2010; revised 16 June 2010; accepted 18 June 2010)

\begin{abstract}
A numerical investigation is performed into the diffusive effects of cylinders positioned in front of a Schroeder diffuser. A regular line of cylinders is shown to offer notable improvements to diffusion from a periodic Schroeder device, provided lateral cylinder spacing is incommensurable with the Schroeder period width. Further investigation considers angular dependence and low frequency results in greater detail, as well as the effects on narrowband and modulated Schroeder devices. An optimization procedure is subsequently performed to investigate the effects of an irregular cylinder arrangement, which provides further diffusive benefits. (C) 2010 Acoustical Society of America.
\end{abstract}

[DOI: $10.1121 / 1.3463817$ ]

PACS number(s): 43.55.Br, 43.20.Fn [LMW]

Pages: $1149-1154$

\section{INTRODUCTION}

Diffusers are well established in room acoustics as a means to treat problems such as echoes while minimising removal of sound energy. ${ }^{1,2}$ Good diffusers contain roughness over different length scales, with the aim of providing predictable spatial and temporal dispersion of sound across a wide bandwidth. Existing designs are almost exclusively surface-based, with the Schroeder diffuser perhaps being the most renowned example. ${ }^{3}$ This comprises a sequence of wells whose depths are determined by a pseudo-random number sequence, thus introducing phase shifts to the reflected sound field.

In this paper the authors consider suspending cylinders in front of a Schroeder diffuser as a means to improve diffuser performance. Previous investigation has suggested potential benefits of such an arrangement, ${ }^{4}$ including improvements to low frequency diffusion and reduction of the effects of 'plate' frequencies (where the Schroeder diffuser acts as a flat plate) associated with designs based on integer number sequences. ${ }^{1}$ This paper aims to provide a more detailed investigation of such diffusive behavior in order to generate effective and practically viable cylinder arrangements.

The use of cylinders as part of a volumetric diffuser ${ }^{5}$ contrasts with the majority of interest in the acoustic properties of cylinder arrays, which has concentrated on sound attenuation or reduced transmission at selected frequencies, ${ }^{6,7}$ although arrays of semicylinders have been employed as surface diffusers. ${ }^{1,8}$ Past efforts to improve the Schroeder design have included modifying the sequence of well depths, fractal embedding of wells within wells, folding of wells to increase effective depth, and modulating adjacent Schroeder diffusers. ${ }^{1}$ All these approaches have relied on altering the diffuser itself, whereas the addition of cylinders would allow retrospective modification of existing installations.

\footnotetext{
a) Author to whom correspondence should be addressed. Electronic mail: markpogson@gmail.com
}

Visual appearance is of key importance to the deployment of diffusers, and the use of cylinders may enhance Schroeder devices in this respect. Furthermore, the space between cylinders and the Schroeder diffuser could be used for a drape system to provide variable room acoustics. ${ }^{9}$ While the addition of cylinders would increase overall diffuser depth, this could be overcome where space is limited by employing a shallower Schroeder diffuser, using sparse cylinder arrangements and only positioning cylinders close to the Schroeder wells. The addition of cylinders is likely to provide advantages over a Schroeder diffuser of the same overall depth, both in terms of improved diffusion and simpler construction.

A periodically repeated Schroeder diffuser is the focus of study due to its widespread practical implementation. Our investigation concentrates on the use of a single line of cylinders, aiming to address issues associated with periodicity in the Schroeder diffuser. Breaking up periodicity appears to offer the greatest potential for improvement; this is supported by evidence that positioning cylinders in front of a single Schroeder device has little positive effect on diffusion. ${ }^{4}$

Scattering arrangements are investigated using a boundary element method (BEM), which is highly accurate and has been validated by practical experiment. ${ }^{1}$ Cylinders are hung parallel to the extruded diffuser wells, hence by assuming infinite lengths of scattering elements, the problem is reduced to two dimensions. ${ }^{10}$

A regular line of cylinders is considered first, investigating the effects of lateral cylinder spacing on diffuser performance. Investigation addresses angular dependence and low frequency effects, as well as scope for improvements to narrowband and modulated Schroeder devices. An optimization procedure is subsequently performed to investigate potential benefits of scattering arrangements with irregular radii and positions of cylinders. Optimization approaches have previously been successfully applied to a variety of diffusers in order to search for design improvements from a range of parameters, ${ }^{1}$ as well as to improve other acoustic properties of cylinder arrays. ${ }^{11}$ The authors use a previously presented 


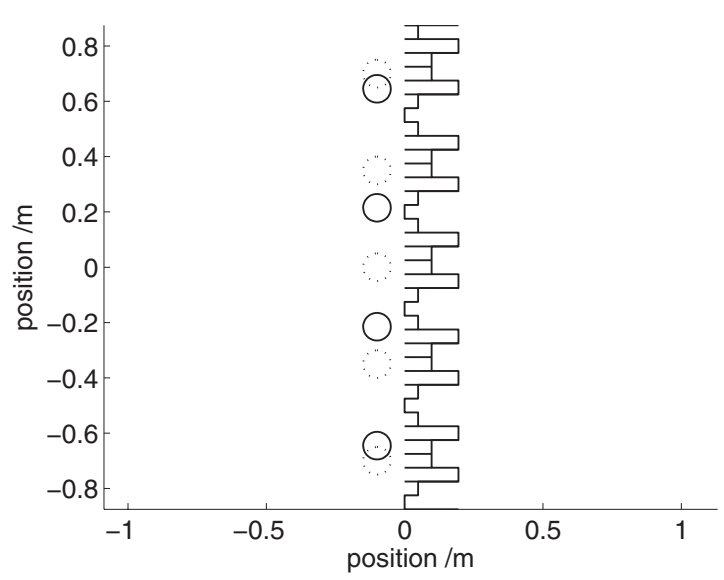

FIG. 1. Schroeder diffuser composed of 5 repetitions of a length 7 QRD, design frequency $500 \mathrm{~Hz}$, with 5 cylinders (dotted circles) and 4 cylinders (solid circles), cylinder radius $5 \mathrm{~cm}$. Cylinder centers are $10 \mathrm{~cm}$ from the Schroeder face.

multiple scattering method ${ }^{4}$ to perform the optimization due to its computational efficiency, with results validated using BEM due to its greater accuracy.

Diffuser performance is assessed in terms of spatial diffusion, with temporal diffusion assumed to be inherent, as is common practice. ${ }^{1}$ Although it is possible for good spatial diffusion to be achieved with poor temporal diffusion, such as with a single cylinder, this can safely be neglected provided scattering arrangements are non-trivial; moreover, the multiple scattering of cylinders in combination with a Schroeder device is likely to provide good temporal diffusion. The diffusion coefficient, an averaged circular autocorrelation of the spatial distribution of scattered pressure, is used to measure diffuser performance. ${ }^{1}$ The diffusion coefficient ranges from 0 to 1 , with better diffusion being indicated by larger values.

\section{REGULAR ARRAYS}

As a starting point for investigation, a regularly spaced line of cylinders is positioned symmetrically in front of a periodic Schroeder diffuser. The Schroeder diffuser is composed of 5 repetitions of a quadratic residue diffuser (QRD) of design frequency $500 \mathrm{~Hz}$; the well depths of each QRD are determined by a quadratic residue sequence of length 7 , well widths are $5 \mathrm{~cm}$ and fins are included between wells, as shown in Fig. 1. Receivers are regularly spaced on a semicircle of radius $10 \mathrm{~m}$, centered on the face of the Schroeder diffuser. A planar source wave is incident normal to the Schroeder diffuser.

Cylinders are of radius $5 \mathrm{~cm}$, positioned $10 \mathrm{~cm}$ in front of the face of the Schroeder diffuser. Spacing between cylinders is varied from $0.15 \mathrm{~m}$ to $1.65 \mathrm{~m}$, with the number of cylinders present for each spacing determined by the total width of the Schroeder diffuser of $1.75 \mathrm{~m}$; two examples of different spacing are shown in Fig. 1. To assess performance, the average of the diffusion coefficient in the frequency range $340-3400 \mathrm{~Hz}$ is calculated; although diffusion can vary greatly with frequency, an average is taken to help sim-

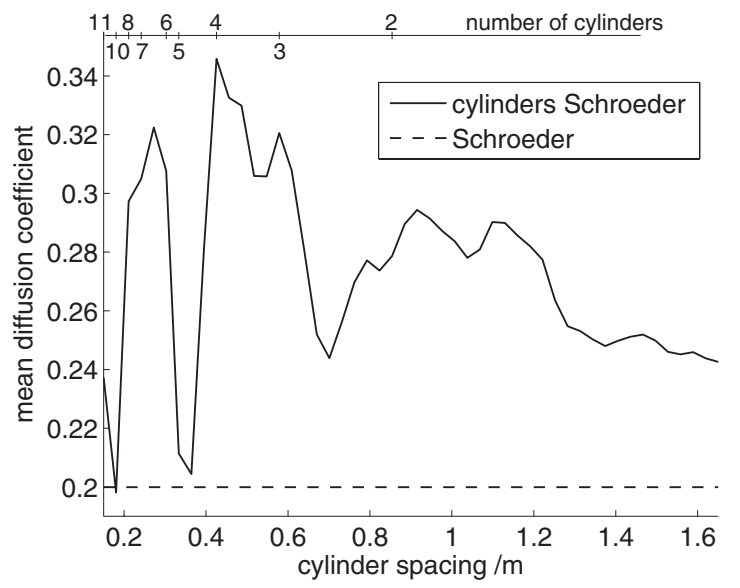

FIG. 2. Mean of the diffusion coefficient in the frequency range 340-3400 $\mathrm{Hz}$ versus spacing between cylinders. The Schroeder period width is $35 \mathrm{~cm}$. The number of cylinders present for different spacing is shown at the top of the graph; this is limited by the total width of the Schroeder diffuser of 1.75 $\mathrm{m}$.

plify data and identify trends. Results are shown in Fig. 2, with the number of cylinders present for different spacing shown at the top of the graph.

Significant improvements to the Schroeder diffuser are evident in Fig. 2. Except for cylinder spacing close to the Schroeder period width and half width $(0.35 \mathrm{~m}$ and $0.175 \mathrm{~m}$ respectively), cylinders have a consistent positive effect on diffusion, increasing the mean diffusion coefficient in the frequency range $340-3400 \mathrm{~Hz}$ by up to around $70 \%$. Even the presence of just two cylinders is able to offer notable diffusive benefits, which could prove particularly useful where space is limited. It is worth noting that if the cylinders are positioned further from the Schroeder diffuser, performance remains very similar, but closer positioning is likely to be more practically desirable. For simplicity, only cylinders are considered in this investigation, but the marked dependence of diffusion on lateral spacing suggests that objects of difference cross-sections may also be able to provide diffusive benefits provided they are appropriately spaced.

In order to further understand the results of Fig. 2, two differently spaced cylinder arrays are considered in greater detail. These are selected to represent the worst and best performance of cylinders according to Fig. 2; one is a line of 5 cylinders spaced according to the Schroeder period width, and the other is a line of 4 cylinders with $43 \mathrm{~cm}$ center-tocenter spacing, as shown in Fig. 1 with dotted and solid lines respectively.

Results for the diffusion coefficient across the frequency range $340-3400 \mathrm{~Hz}$ for each arrangement, as well as for the Schroeder diffuser alone, are shown in Fig. 3; the result without cylinders for a flat plate of the same total width is also displayed to provide a reference as a non-diffusing surface.

As suggested by Fig. 2, the 5 cylinder array has little effect on the quality of diffusion - the commensurable spacing of cylinders means that periodicity in the Schroeder diffuser is unaffected. In contrast, the 4 cylinder array has a consistent positive effect, which is attributable to the nonsimple relation between the period width of the Schroeder 


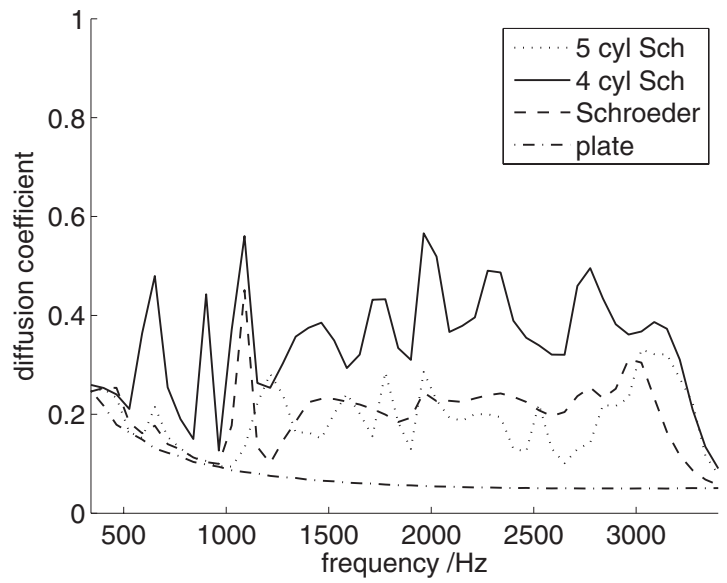

FIG. 3. Diffusion coefficients for the arrays shown in Fig. 1, including results for the Schroeder diffuser alone and a flat plate of the same width.

diffuser and the center-to-center spacing of cylinders. Improvements due to the 4 cylinder array appear fairly uniform across the frequency range.

At around $3500 \mathrm{~Hz}$, where the Schroeder diffuser behaves as a flat plate due to all wells re-radiating in phase, ${ }^{1}$ little benefit is provided by the cylinders, since performance here is unaffected by periodicity, hence there is little scope for improvement by the regular line of cylinders; however, multiple layers of cylinders, or staggering cylinders relative to the Schroeder diffuser, might be able to address this (as considered later with regard to optimization). Behavior below around $1000 \mathrm{~Hz}$, where the Schroeder device provides little diffusion, is improved by the 4 cylinder array, and will be considered further below.

It is worth noting that the lateral spacing of cylinders is key to the diffusive effect: if the 5 cylinder array were shifted such that it were no longer centered on the Schroeder diffuser, or the lateral spacing of the 4 cylinder array were made equal to the Schroeder period width, similar results would be obtained as for the 5 cylinder arrangement in Fig. 3 (results not shown).

As another measure of performance, a polar plot of scattering is shown in Fig. 4. Sound pressure levels of scattering are displayed for a $2500 \mathrm{~Hz}$ source wave at normal incidence to the 4 cylinder arrangement in Fig. 1, as well as for the Schroeder diffuser alone. The reduction of grating lobes by the cylinder array is evident in the polar plot, particularly

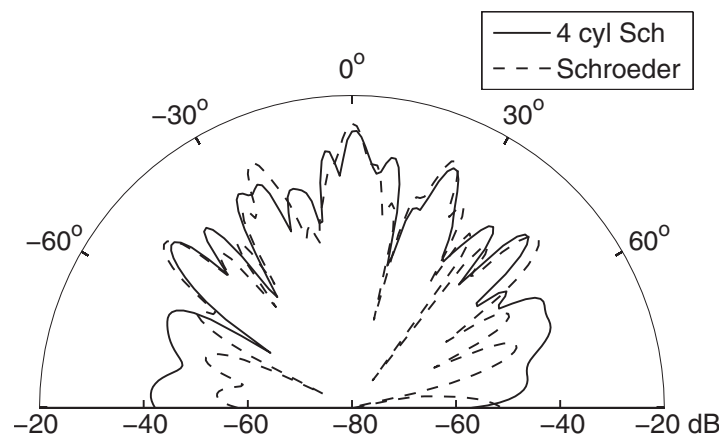

FIG. 4. Polar plot of scattering at $2500 \mathrm{~Hz}$ for 4 cylinders with Schroeder diffuser (solid line) as shown in Fig. 1, and Schroeder alone (dashed line).

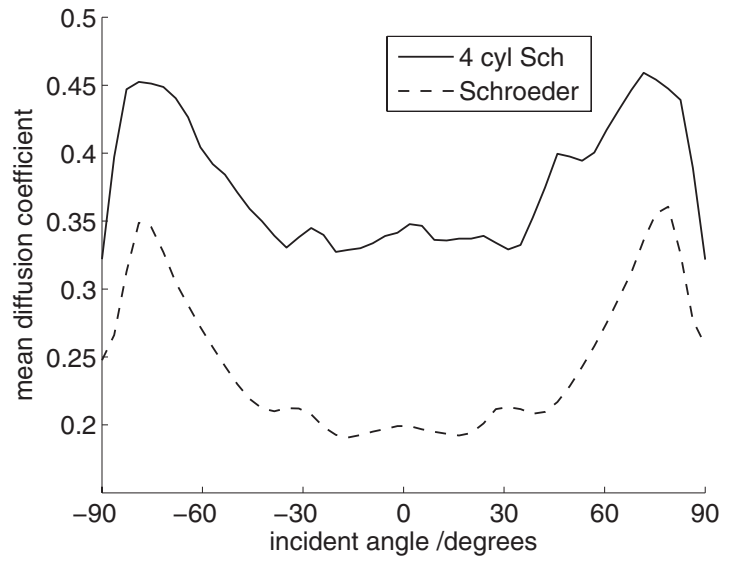

FIG. 5. Mean of the diffusion coefficient in the frequency range 340-3400 $\mathrm{Hz}$ versus the angle of incidence, where $0^{\circ}$ is normal to the diffuser.

around $-60^{\circ}, 15^{\circ}, 40^{\circ}$ and $80^{\circ}$. Scattering amplitude at oblique angles is also increased. From Fig. 3 it appears that this improvement is fairly representative of the benefits provided by the 4 cylinder array.

Although diffusers are frequently positioned normal to the incident sound, this is not always the case: devices are sometimes positioned on side walls or at an angle, while a variety of source locations might occur, and reflections from different directions may be significant; a good diffuser should therefore perform well over a range of incident angles.

To assess angular dependence, the 4 cylinder arrangement shown in Fig. 1 is again used, and the diffusion coefficient between $340-3400 \mathrm{~Hz}$ is averaged for different angles of incidence, as shown in Fig. 5. The positive effect of cylinders appears consistent across all angles, displaying a very similar angular dependence to the Schroeder diffuser alone. This is because the main effect of cylinders is to counteract periodicity in the Schroeder diffuser, which is unaffected by incident angle.

Low frequency diffusion is a key aspect of diffuser design, as space constraints often limit performance. The 4 cylinder array in Fig. 1 is used as a basis for further investigation of the effect of cylinders on low frequency diffusion from the Schroeder diffuser. Of interest is the effect of cylinder size and the distance between the Schroeder diffuser and cylinders. The quality of diffusion is assessed by averaging the diffusion coefficient in the range $340-1040 \mathrm{~Hz}$ (wavelengths 1-0.33 m), where the Schroeder diffuser alone provides little diffusion (as evident in Fig. 3). The results of varying cylinder radius and separation between the Schroeder diffuser and cylinders are shown in Fig. 6.

Low frequency diffusion improves steadily with increasing cylinder radius up to around $10 \mathrm{~cm}$, due to larger cylinders providing greater scattering amplitude at low frequency, hence having a greater effect on diffusion. To provide low frequency improvements to the Schroeder diffuser, cylinders must therefore be sufficiently large: to significantly scatter sound $^{12}$ at wavelength $\lambda$, cylinder radius $a \geqslant \lambda / 2 \pi$. The result tends to level off for larger radii in Fig. 6, which is due to the diffusion coefficient being averaged over a range of frequencies - if a lower frequency upper limit were used, 


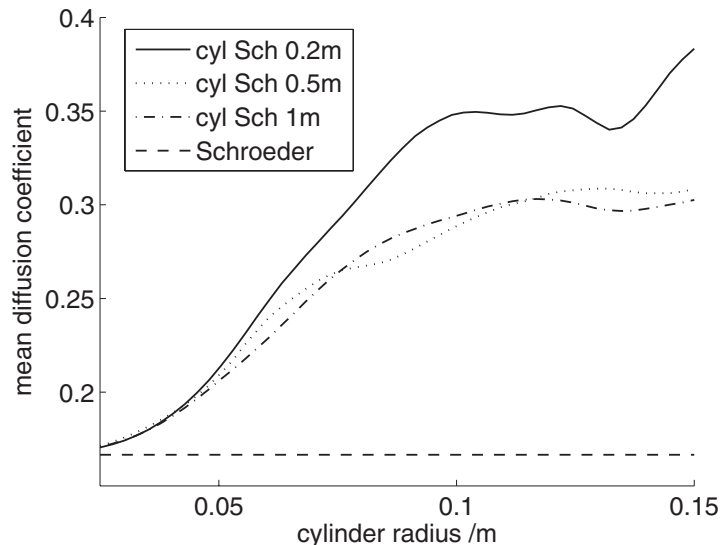

FIG. 6. Mean of the diffusion coefficient in the frequency range 340-1040 $\mathrm{Hz}$ (wavelengths $1-0.33 \mathrm{~m}$ ) versus cylinder radius, based on the 4 cylinder arrangement in Fig. 1. Results are shown for 3 different distances between the cylinders and Schroeder diffuser $(0.2 \mathrm{~m}, 0.5 \mathrm{~m}$ and $1 \mathrm{~m})$, as well as for the Schroeder diffuser alone.

the leveling off would shift to a larger cylinder radius (results not shown). A small fluctuation in low frequency diffusion is observed for the largest cylinder radius with the smallest separation between cylinders and the Schroeder wells, which is presumably due to the proximity of the cylinder surface to the Schroeder well resulting in less predictable scattering behavior.

Results suggest that low frequency diffusion does not increase with the separation between the Schroeder diffuser and cylinders; in fact, moving cylinders further away from the Schroeder diffuser seems to reduce low frequency diffusion due to the weaker coupling between cylinders and wells resulting in a smaller diffusive effect. This implies that improvements to low frequency diffusion by the cylinders is not due to multiple scattering paths effectively increasing the depths of the Schroeder wells, but simply through breaking up periodicity: at low frequency, diffusion from the Schroeder device in this instance is limited by periodicity rather than depth, ${ }^{1}$ and the reduction of the effects of periodicity by the cylinders appears to be the reason for low frequency improvements.

\section{REGULAR ARRAYS WITH OTHER SCHROEDER DIFFUSERS}

Only a single type of Schroeder diffuser has been considered so far. Of interest is the effect of cylinders on other Schroeder devices, including one designed to perform over a narrower bandwidth, which due to its smaller size might be implemented where space is limited. To investigate this, a Schroeder diffuser is composed of 5 repetitions of a length 7 QRD, similar to previously, but with design frequency 1500 $\mathrm{Hz}$ and well widths $3 \mathrm{~cm}$. The cylinder array is based on the 4 cylinders in Fig. 1 but with $30 \mathrm{~cm}$ lateral spacing due to the smaller total width of the Schroeder diffuser. Diffusion coefficient results are shown in Fig. 7. Improvements to the Schroeder device by the cylinders are very similar to previously, including a reasonable enhancement to diffusion below the design frequency.

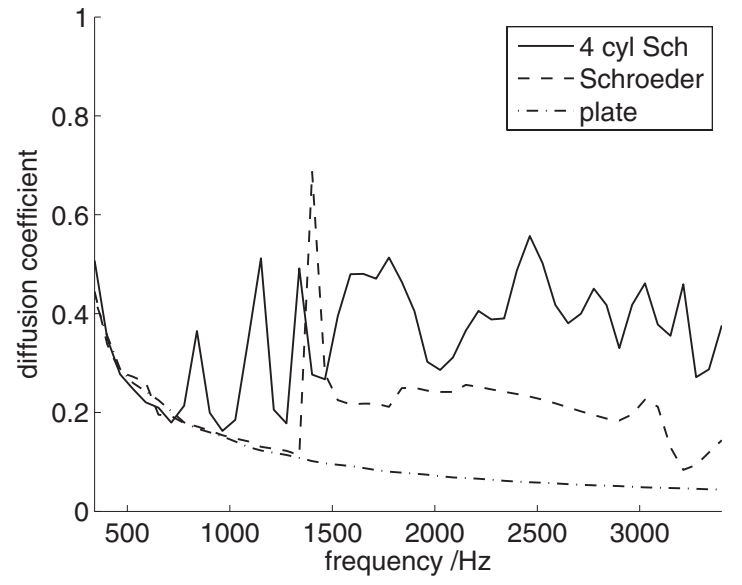

FIG. 7. Diffusion coefficients for array similar to the 4 cylinder arrangement in Fig. 1, but with Schroeder well widths $3 \mathrm{~cm}$ and design frequency 1500 $\mathrm{Hz}$, and lateral cylinder spacing $30 \mathrm{~cm}$.

The main contribution of cylinders appears to be in tackling problems associated with periodicity in the Schroeder diffuser. This has been addressed in the past by modulating different Schroeder base shapes to form larger devices, ${ }^{13}$ and it is of interest whether cylinders are able to provide further improvements to such a design. As a basis for investigation, a length 7 QRD (A) of design frequency $500 \mathrm{~Hz}$ is modulated with a length $5 \mathrm{QRD}(\mathrm{B})$ of the same depth to form a sequence ABBABA. ${ }^{1}$ The same 5 cylinder array as in Fig. 1 is centered $25 \mathrm{~cm}$ in front of the modulated Schroeder device, and diffusion coefficients for the arrangement are plotted in Fig. 8.

The presence of cylinders again improves performance, albeit less dramatically; the average diffusion coefficient is 0.36 , compared with 0.25 for the Schroeder diffuser alone. Although the spacing of the 5 cylinders was previously not beneficial to diffusion (due to being commensurable with the Schroeder period), the changes to the Schroeder diffuser mean this is no longer the case, despite the length 7 QRDs in the modulation having the same width as used previously. However, performance fluctuates quite markedly across the frequency range, which appears to be due to the modulation

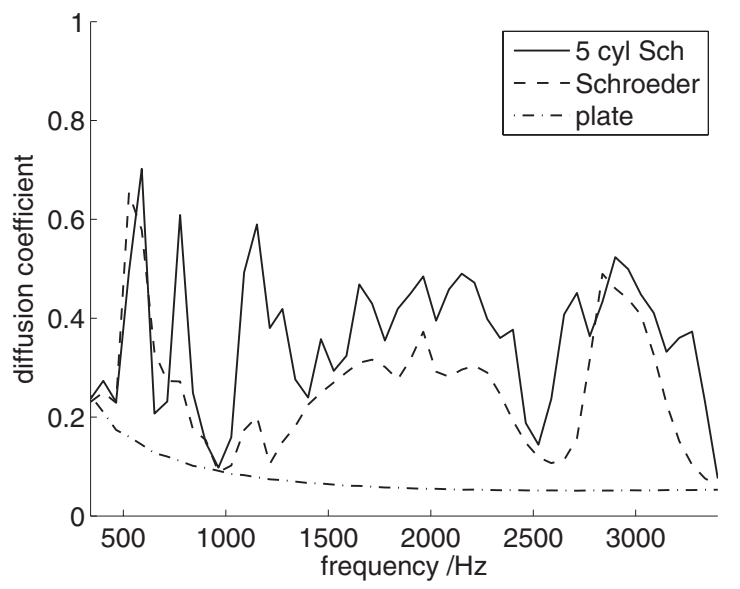

FIG. 8. Diffusion coefficients for array similar to the 5 cylinder arrangement in Fig. 1, but with the Schroeder diffuser composed of a length 7 (a) and length 5 (b) QRD modulated to form a sequence ABBABA. The 5 cylinder array is spaced $25 \mathrm{~cm}$ from Schroeder face. 


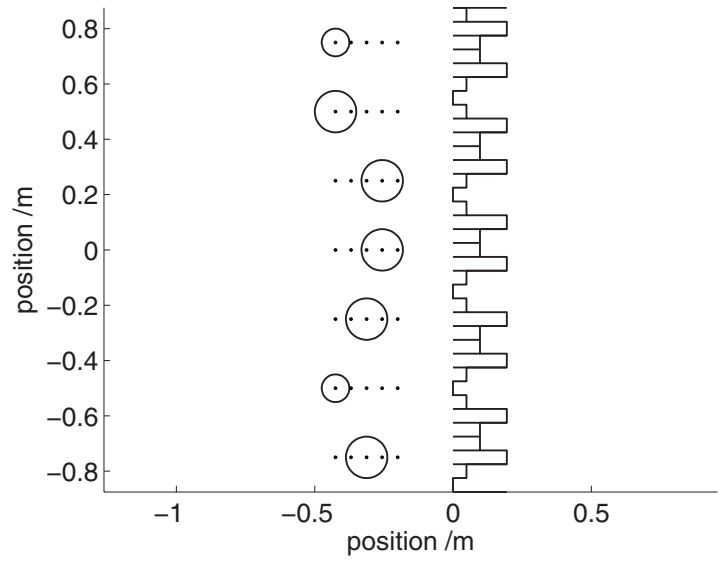

FIG. 9. Cylinder array generated by genetic algorithm. Grid points for possible positions are shown by dots. Cylinders are of radius $5 \mathrm{~cm}$ and $7.5 \mathrm{~cm}$.

of the Schroeder diffuser affecting the frequencies at which the regularly spaced cylinders are able to improve diffusion. This may correspond with the fact that spacing the cylinders at $25 \mathrm{~cm}$ rather than $10 \mathrm{~cm}$ from the Schroeder diffuser provides better performance, which did not appear to be the case for periodic devices.

\section{OPTIMIZATION}

The introduction of irregular cylinder radii and positions should benefit diffusion by increasing roughness over a range of length scales, thus providing more random scattering from cylinders over a variety of frequencies. While it is not possible to evaluate systematically all combinations of effects, an optimization procedure is able to search in an automated manner for favorable arrangements from a number of parameters. ${ }^{14}$ In order to simplify investigation, cylinders are restricted to a grid with only a discrete set of possible radii; not only does this make the optimization procedure more straightforward by reducing the size of the search space, it also means results would be more practically viable to construct.

A genetic algorithm is the most appropriate method of optimization due to the discrete nature of arrangements, and a standard procedure is implemented. ${ }^{1}$ The performance of each array is assessed in terms of a single metric, which is selected as the mean of the diffusion coefficient for normal incidence over the range $340-3400 \mathrm{~Hz}$ minus the standard deviation, thus reflecting both overall effectiveness and uniformity of diffusion. There will realistically be several similarly good results, and a global maximum is very unlikely to be found, so in practice the algorithm is only expected to obtain reasonable diffusion results. This is especially noteworthy because by imposing particular grid positions and radii, it means that even if a global maximum were found for those particular constraints, the maximum would only be for a fairly arbitrary configuration. For convenience and applicability to existing diffusers, Schroeder well depths are not optimized by the genetic algorithm.

An array of grid points is positioned symmetrically in front of 5 repetitions of a length 7 QRD, as shown in Fig. 9. Grid positions may be either empty or occupied by a cylinder

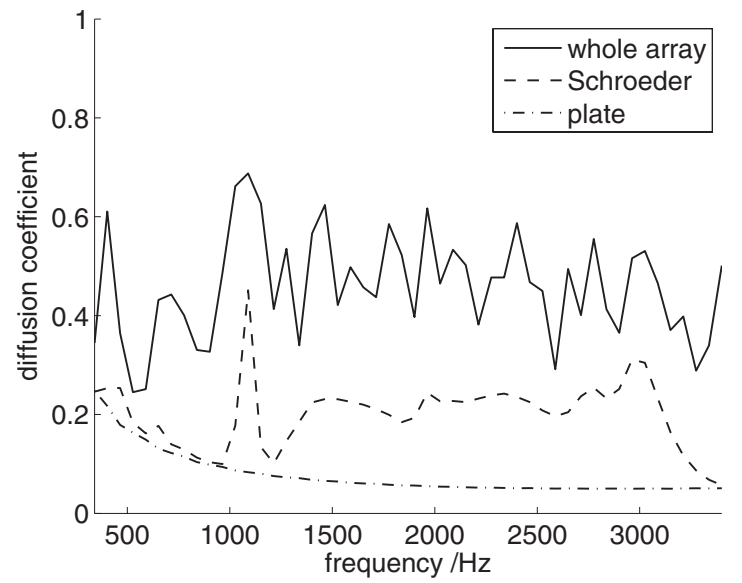

FIG. 10. Diffusion coefficients for result from genetic algorithm in Fig. 9.

of radius $2.5,5$ or $7.5 \mathrm{~cm}$. The grid is composed of 7 rows (each running perpendicular to the Schroeder face) and 5 columns. Spacing between rows is $25 \mathrm{~cm}$, which is incommensurable with the Schroeder period width; based on Fig. 2 , this spacing should provide good diffusion, and offers more scope for optimization than the $43 \mathrm{~cm}$ spacing considered previously, which would only allow space for 4 rows. Each row may contain at most 1 cylinder, meaning no cylinder is obscured. Columns are separated by $5.62 \mathrm{~cm}$, and lie between $20 \mathrm{~cm}$ and $42.48 \mathrm{~cm}$ from the Schroeder face. The setup is intended to provide a single-layer cylinder array which includes variation of cylinder radii and positions.

The genetic algorithm is performed using a multiple scattering approximation, ${ }^{4}$ and results are validated using BEM. A successful array is shown in Fig. 9; worth noting is that all rows have cylinders, and only the two largest cylinder radii are present. Corresponding diffusion coefficient results obtained using BEM are shown in Fig. 10. The diffusion coefficient for the whole array is compared against that for the Schroeder diffuser alone and a flat plate of the same width.

The optimized sequence of cylinders provides considerable improvements to diffusion across the frequency range, with a mean diffusion coefficient of 0.46 ; this provides a $130 \%$ increase over the result for the Schroeder diffuser alone, and an increase of around 35\% over the best regular array spacing in Fig. 2. Although some larger cylinders are present in the optimized array than in Fig. 2, this alone does not account for the increase in diffusion; rather it is the combination of different positions and radii. Perhaps most significant is the removal of the Schroeder plate frequency at around $3500 \mathrm{~Hz}$; the irregularity of the array means the cylinders are able to address issues other than just those associated with periodicity in the Schroeder diffuser. Nonoptimized arrays can provide diffusive benefits approaching those of the optimized array; results are not shown for simplicity, but a good example is obtained by staggering cylinders on the grid points shown in Fig. 9 according to a quadratic residue sequence.

To ensure that results are not 'over-fitted', ${ }^{1}$ where arrays only perform well for the conditions used to rank them in the genetic algorithm rather than being good diffusers in a 


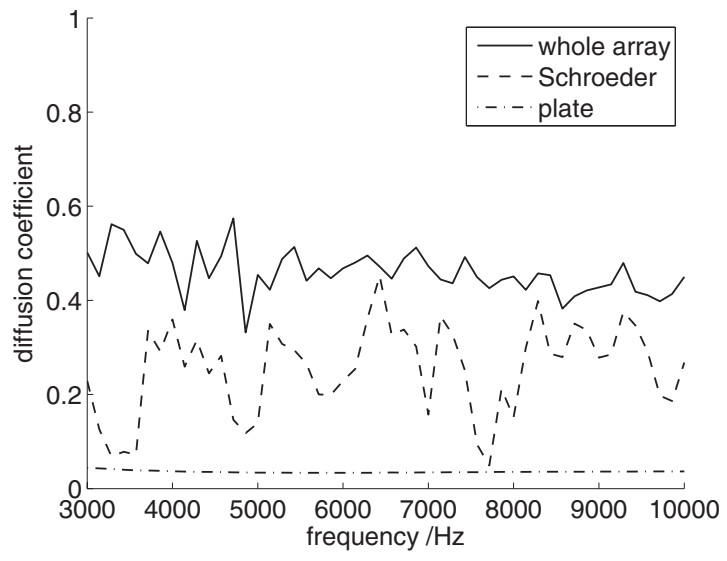

FIG. 11. Diffusion coefficients for result from genetic algorithm in Fig. 9, with source wave incident at $45^{\circ}$ across frequency range $3-10 \mathrm{kHz}$.

broader context, the array is tested with different angles of incidence and frequencies, suggesting that the result is robust; an example is shown in Fig. 11, which demonstrates stronger and more uniform diffusion across frequencies with the optimized arrangement than for the Schroeder diffuser alone.

\section{CONCLUSION}

Positive results have been obtained for the diffusive effects of cylinders positioned in front of a Schroeder diffuser, which could be straightforwardly applied or retrofitted to existing devices. The effects of cylinders appear uniform across different angles of incidence. Cylinders are able to provide improvements to diffusion at low frequencies, provided cylinder radii are sufficiently large to cause significant scattering. Further to improving diffusion, cylinders may also provide aesthetic advantages.

Clear diffusive benefits are demonstrated with a very simple line of cylinders in front of a periodic Schroeder device, provided cylinder spacing is incommensurable with the Schroeder period width. Cylinders also offer benefits to narrowband and, to a lesser extent, modulated Schroeder devices, with increases to the diffusion coefficient in the frequency range $340-3400 \mathrm{~Hz}$ typically at least around $50 \%$.
Further improvements are obtained by an optimization method, including the removal of Schroeder plate frequencies, which demonstrates the benefits of staggering cylinders of different sizes, although the simplicity of an incommensurable row of regularly spaced cylinders may be most promising in terms of practical implementation.

\section{ACKNOWLEDGMENTS}

This work was funded by the UK Engineering and Physical Sciences Research Council (EPSRC), under Grant No. EP/D031621.

${ }^{1}$ T. J. Cox and P. D'Antonio, Acoustic Absorbers and Diffusers, 2nd ed. (Spon, London, 2009), pp. 289-330.

${ }^{2}$ H. Kuttruff, Room Acoustics, 4th ed. (Spon, London, 2000), pp. 55-58.

${ }^{3}$ M. R. Schroeder, "Binaural dissimilarity and optimum ceilings for concert halls: More lateral sound diffusion," J. Acoust. Soc. Am. 65, 958-963 (1979).

${ }^{4}$ M. Pogson, D. M. Whittaker, G. A. Gehring, R. J. Hughes, J. A. S. Angus, and T. J. Cox, "Multiple scattering between cylinders and a Schroeder diffuser," Acta. Acust. Acust. 96, 292-299 (2010).

${ }^{5}$ T. J. Cox, R. J. Hughes, J. A. S. Angus, D. M. Whittaker, M. Pogson, and G. A. Gehring, "Volumetric diffusers inspired by percolation fractals," in Proceedings of the Institute of Acoustics (2008), Vol. 30.

${ }^{6}$ O. Umnova, K. Attenborough, and C. M. Linton, "Effect of porous covering on sound attenuation by periodic arrays of cylinders," J. Acoust. Soc. Am. 119, 278-284 (2006).

${ }^{7}$ J. V. Sánchez-Pérez, D. Caballero, R. Martinez-Sala, C. Rubio, J. SanchezDehesa, F. Meseguer, J. Llinarez, and F. Galvez, "Sound attenuation by a two-dimensional array of rigid cylinders," Phys. Rev. Lett. 80, 5325-5328 (1998).

${ }^{8}$ P. Boulanger, K. Attenborough, Q. Qin, and C. M. Linton, "Reflection of sound from random distributions of semi-cylinders on a hard plane: Models and data," J. Phys. D: Appl. Phys. 38, 3480-3490 (2005).

${ }^{9}$ P. D'Antonio and T. J. Cox, "Diffusor application in rooms," Appl. Acoust. 60, 113-142 (2000).

${ }^{10}$ T. J. Cox, "Predicting the scattering from reflectors and diffusers using two-dimensional boundary element methods," J. Acoust. Soc. Am. 96, 874-878 (1994).

${ }^{11}$ A. Håkansson, F. Cervera, and J. Sánchez-Dehesa, "Sound focusing by flat acoustic lenses without negative refraction," Appl. Phys. Lett. 86, 054102 (2005).

${ }^{12} \mathrm{P}$. M. Morse and K. U. Ingard, Theoretical Acoustics (Princeton University Press, Princeton, NJ, 1986), pp. 400-405.

${ }^{13}$ J. A. S. Angus, "Using grating modulation to achieve wideband large area diffusers," Appl. Acoust. 60, 143-165 (2000).

${ }^{14}$ J. Nocedal and S. J. Wright, Numerical Optimization (Springer, New York, 2006), pp. 1-3. 